\title{
Platinum nanoparticle in tantalum electrode for the electrochemical analysis of heavy metal ions formed by the ion beam sputtering deposition
}

\author{
Ahmad Khalaf Alkhawaldeh* \\ University of Jordan, Department of Chemistry, Amman - 11942, Jordan. \\ *E-mail: Ahm9140096@fgs.ju.edu.jo, Ahmad.alkawalda@yahoo.com \\ Phone: 00962787455167
}

\begin{abstract}
Today, contamination from heavy metals in the atmosphere is a global concern. Efficient detection techniques are therefore necessary if heavy metal exposure levels in different media are to be determined. The voltammetry method for in situ detection of heavy metal ions is a very sensitive electrochemical method. This thesis explores emerging developments in electrode alteration, materials production and experimental optimization. An electrochemical sensing platinum nanoparticle in the tantalum electrode is provided by means of an Ion Beam Sputtering Deposition (IBSD). The electrode was made with a Pt solution, sputtered simultaneously with hydrochloric acid corrosion on tantalum substrate. In the study of heavy metal ions, for example, the platinum nanoparticle electrodes as prepared were used Square wavelength voltammetry (OSWV) $\mathrm{Hg}^{2+}, \mathrm{Cu}^{2+}$ and $\mathrm{Ag}^{2+}$. The porous electrodes were observed in a broader range by the Pt nanostructure electrode for heavy metal ions. Furthermore, the susceptibility to detection has been shown to be saturated as the thickness of the layer electrode exceeded $50 \mathrm{~nm}$. For $\mathrm{Hg}^{2+}$ 0,003-1 $\mathrm{M}$, for $\mathrm{Cu}^{2+} 0,005-3 \mathrm{M}$ and for $\mathrm{Ag}^{2+}$ the linear detection scale is 0,009-4 M. There has also been good reusability and repeatability. In addition, a scan electron microscope (SEM) used to study platinum electrode forming process and nanostructure. This electrode will have interesting applications in sensing systems.
\end{abstract}

Key Words: Heavy metal, Platinum electrode, Ion beam sputtering deposition, Nanoparticle, IBSD, Ion detection.

\section{Introduction}

Due to its high degradability, aggregation in live species, which cause diverse diseases and genetic abnormalities, and deleterious ecological consequences, heavy metal ions pose a major threat to human health $[1,2]$. First, $\mathrm{Pb}^{2+}$ can lead to a wide variety of health conditions, including diarrhea, convulsions, vomiting, and insufficiency of the kidneys, tumors and negative metabolism. [3]. Excessive $\mathrm{Cu}^{2+}$ can also be blamed for other illnesses, including hemochromatosis, catarrh, calf cramps, scalp dermatitis brass chills and Wilson's [4]. Several methods have been employed for detecting heavy metal ions, for instance, the use of atomic electron emission spectrometry (ICP / AES, ICP / 
MS), flow-injection spectroscopy (FIAAS), and high resolution liquid chromatography (HPLC) [5-7]. However, where the concentration spectrum of these metal ions is wide, it is difficult to examine specifically heightened metal ions using these techniques. Therefore, it is urgently necessary to build metal ion sensors with a broader range of detections and high sensitivity. Graphene is a single-atomic layer of tightly packed carbon atoms in a two-dimensional wave $[8,9]$. Due to its high electronic mobility, wide surface area and the prevalence of exposed edge planes for greatly raising the storage of charges, platinum has tremendous potential to be used in the production of chemo sensors [10]. The wide range of catalytic, sensor and plasma resonance applications have drawn tremendous attention for porous substances, such as porous gold with a high volume-tosurface ratio, strong electrical and thermal conductivity and a high catalytic performance $[11,12]$. Porous gold has recently been shown to be a highly efficient catalyst for many primary reactions such as low-temperature $\mathrm{CO}$ oxidation, electrical oxidation, and reduction of oxygen-based hydrogen peroxide [13]. A variety of methodologies have been studied to prepare pore structures, including wet impregnation, relocation, solvent fusion and particulate leaching, rough anodization and magnetron sputtering [14]. Over the years significant advances have taken place in electrochemical science, including the modification of the various types of solid electrodes now being used to test specific sample forms [15]. Recently, the low cost, flexibility and fast adsorption of inorganic materials have drawn significant interest [16]. The most widely used material in electrochemical measurement of heavy metals, particularly nanomaterial-based metals, metal oxides, carbonated metals and their composites [17]. The specific feature of the Ion beam sputtering (IBSD) process is the capacity to deposit high density films at a low substratum temperature through the dynamics for atomic or molecular transport growth mechanism, which gives it numerous benefits including excellent stoichiometry movement, strong durability and simple set-up for the creation of films [18-20]. The platinum nanoelectrode was rendered in tantalum surface by IBSD and a novel electrode was then used for electrochemical study for detecting of heavy metal ions $\left(\mathrm{Hg}^{2+}, \mathrm{Cu}^{2+}\right.$ and $\mathrm{Ag}^{2+}$ ) in the different mediums.

\section{Materials, Apparatus and Detection of metal ions}

Shandong Laiyang Economic and Technologi are bought with hydrochloric acid (37\%), neritic acid (98\% AR), ammonium acetate (AR), persulfate potassium (AR), oxide phosphate (V) (98\%), permanganate potassium (AR), peroxide hydrogen (30\%), ethanol (AR), sodium nitrate (AR), and diethyl ether (AR). The Tianjin Aida Hengsheng Manufacturing was acquired with platinum electrode fittings (diameter $6 \mathrm{~mm}$ ), platinum foil auxiliary and $\mathrm{Ag} / \mathrm{AgCl}$ reference electrodes. Zhuhai Kaivo Electronic Components purchased tantalum tiles. Qingdao Heli Gas bought ultra-pure nitrogen (99.999\%). Flam's ultra-pure water device was developed. Images from a scan-electron microscope were obtained using a JEOL JSM-6700F electron-scan microscope with an accelerated voltage of $5 \mathrm{kV}$. An EDS detector attached to the above-mentioned SEM microscope, Oxford INCA Sight-X, has been used to carry out energy dispersion testing (EDS). The 
concustomized three-electrode cell with the electrochemical workstation CHI-760D (CHI Devices, Shanghai, China) was used to perform all electrochemical measurements. At least 15 minutes prior to data collection, the solution had been degassed with nitrogen and blanketed with nitrogen over the completely experimental phase. At room temperatures, all capacity is reached in an electrical one-compartment cell. The IBSD was made of platinum nanoparticle electrode. The electrodes were cleaned thoroughly and dried in oven prior to use with ultra-pure water.

The Square Wave Voltammetry of Oster young (OSWV) has been used in order to illustrate realistic applications of the platinum nanoparticle electrode, to evaluate $\mathrm{Hg}^{2+}$, $\mathrm{Cu}^{2+}$ and $\mathrm{Ag}^{2+}$ solutions that are more sensitive than differential pulse voltammetry because of the improved reverse reaction sensibility. A mixture of a square wave series with an escalator waveform is the ideal waveform for OSWV. On each square-wave period, the current is sampled twice, one at the end of the forward pulse and one at the end of the opposite pulse. $\mathrm{Hg}^{2+}, \mathrm{Cu}^{2+}$ and $\mathrm{Ag}^{2+}$ solutions were reduced in stock solutions at various rates. The electrodes were washed in an ammonium acetate buffer to ensure complete separation of all the ions with OSWV in transparent solutions of an ammonium acetate buffer.

\section{Results and discussion}

The composition and the cross-section of the electrodes is characterized by the use of SEM testing to ensure the effectiveness of the platinum nanoparticle on the tantalum composition [21]. Figure 1 shows that the electrode is flat and Pt is compacted very tightly. Nevertheless, after hydrochloric corrosion, the microstructure was obtained and pore is distributed similarly. It is very uniform in its dimensions and has a diameter of around $40-75 \mathrm{~nm}$. 


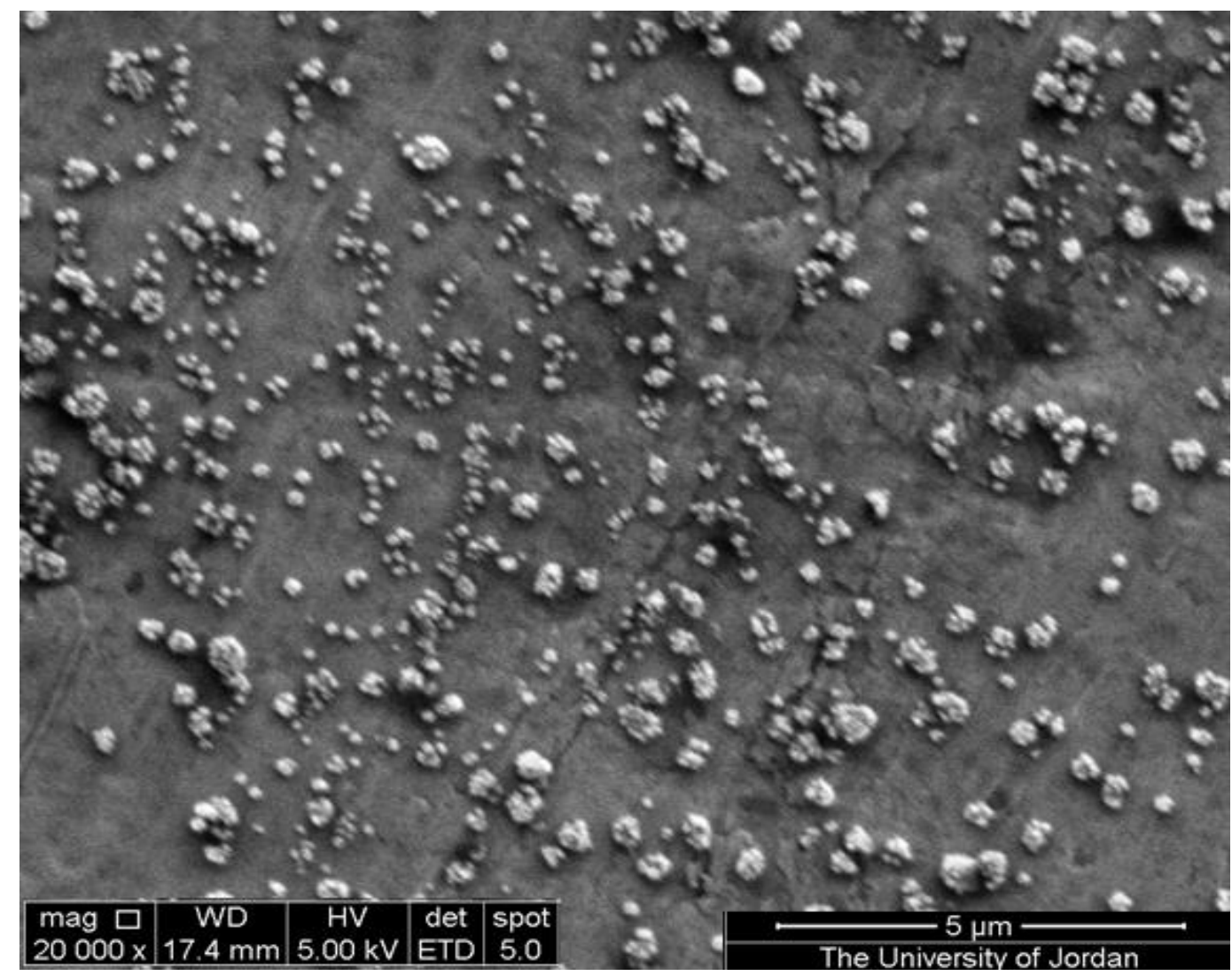

Figure 1; SEM image of platinum nanoelectrode coated on tantalum substrate.

In the preparation of the composite target on Ta top, SEM was used to describe the platinum nanoelectrode. In about $150 \mathrm{~nm}$ to $1 \mathrm{~m}$ of the SEM picture, the individual platinum surface was nearly translucent. The thickness of this $\mathrm{Pt}$ nanoelectrode is relatively uniform, and about $32 \mathrm{~nm}$ in diameter, as seen in Figure 1. 


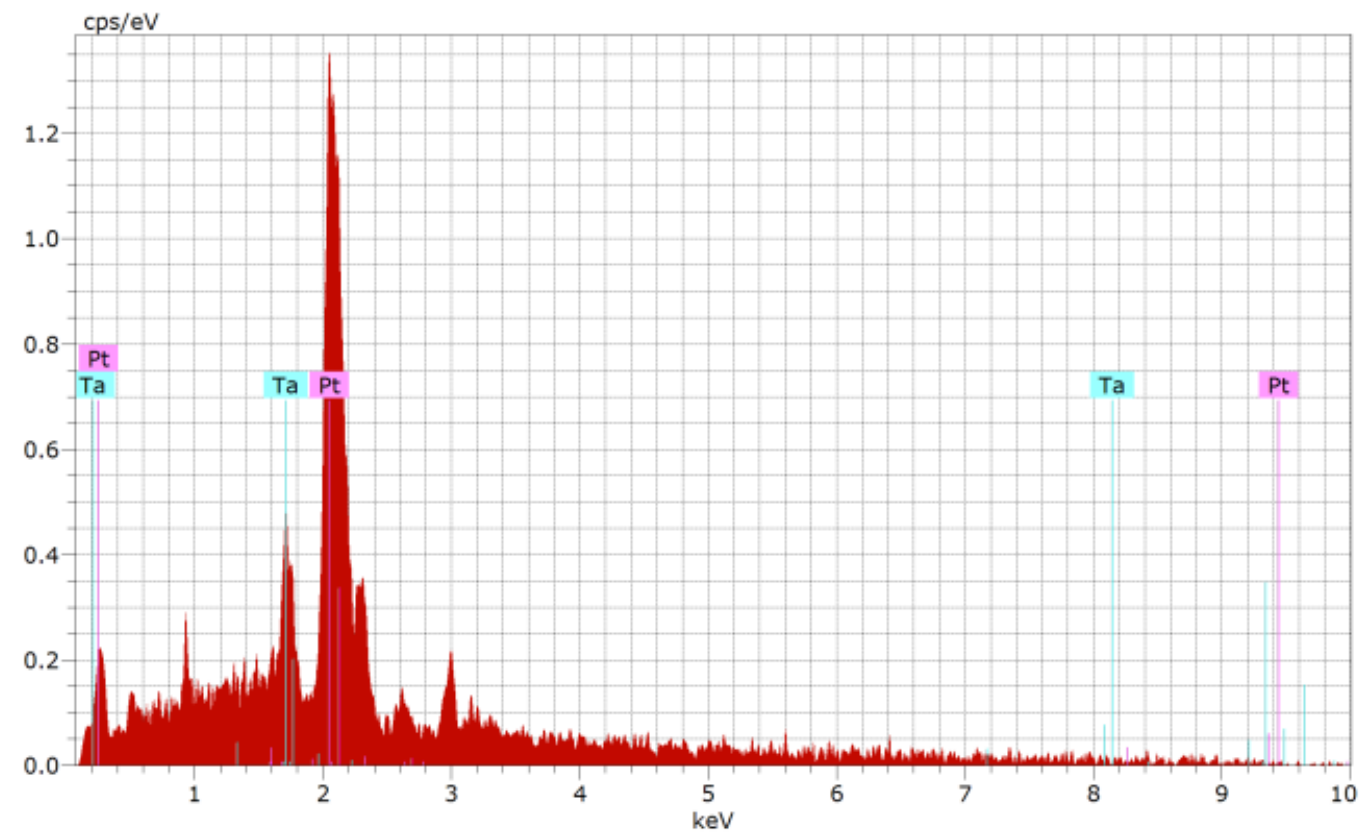

Figure 2; EDX image of platinum nanoelectrode coated on tantalum substrate.

An EDX spectrum of deposited particles further confirms the identity of the platinum nanoparticles deposited on a tantalum substrate (Figure 2). Platinum and tantalum are the main peaks of the EDX spectrum.

One of the most important research techniques for metal ions identification in natural, health and industrial samples is electrochemical research. Possibly, due to special Pt nanostructures and the modern tantalum coating, the electrochemical calculation using platinum nanoelectrode shows enhanced detection range and greatly increased the real area of the electrode allowing more metal ions to react to the electrode. 


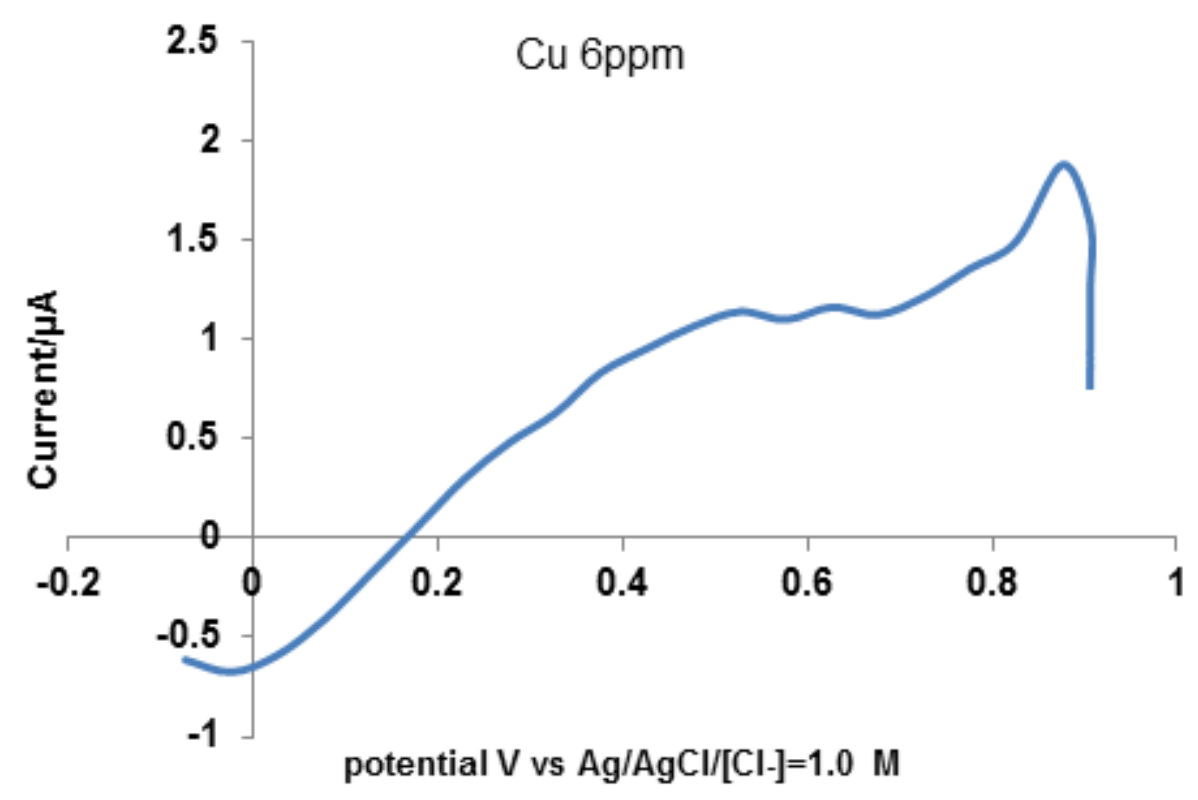

Figure 3; cyclic voltammograms of $\mathrm{Cu}^{2+}$ solutions with $6 \mathrm{ppm}$ concentration using the Platinum nanoelectrode at a scan rate of $50 \mathrm{mV} \mathrm{s}^{-1}$.

As shown in Figure 3, the platinum nanoelectrode detects $\mathrm{Cu}^{2+}$ with a buffer solution for ammonium acetate $(\mathrm{pH}=5)$, that is used as a model analytic. Since Oster young square wave voltammetry (OSWV) typically has greater analytical sensitivity, all detections were performed using OSWV in the sample for all heavy metal ions. $\mathrm{Cu}^{2+}$ solutions with various rates have been reported from $0.0 \mathrm{~V}$ through $0.38 \mathrm{~V}$ in a potential window. The prominent peak range from $0.81-0.92 \mathrm{~V}$ to the reference electrode $\mathrm{Ag} / \mathrm{AgCl}$ has been described to deliver well-defined peaks [22]. 


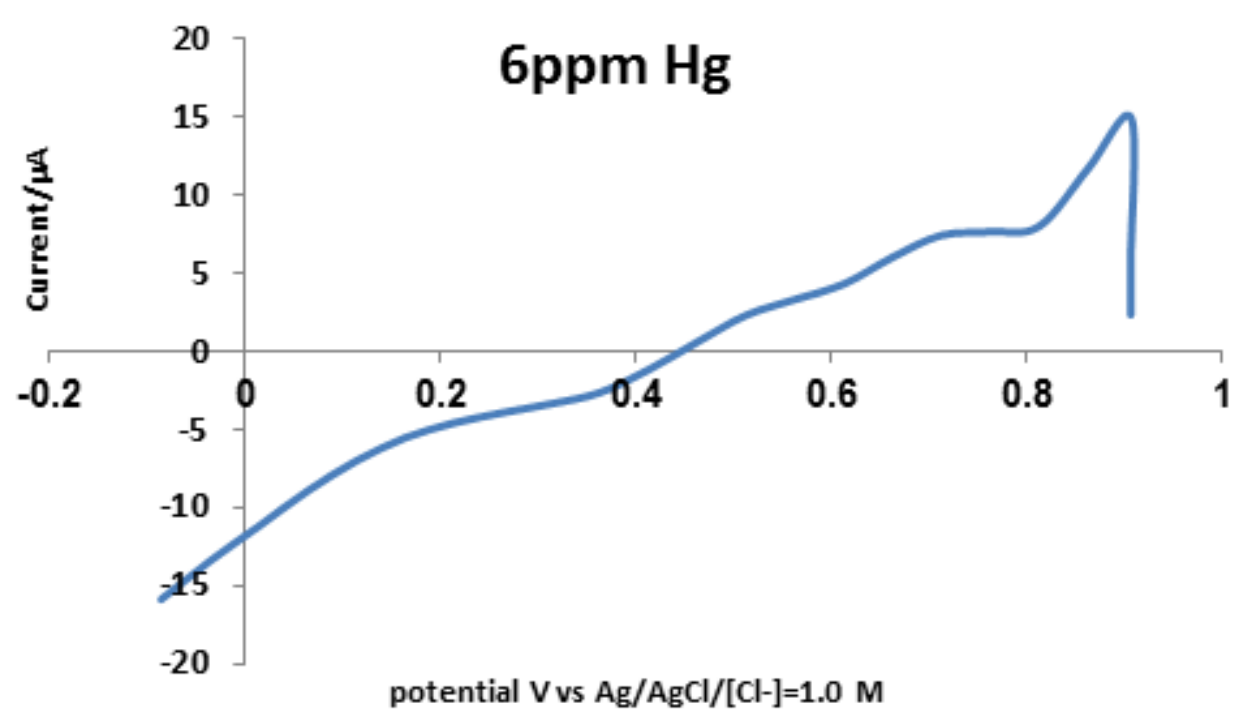

Figure 4; cyclic voltammograms of $\mathrm{Hg}^{2+}$ solutions with $6 \mathrm{ppm}$ concentration using the Platinum nanoelectrode at a scan rate of $50 \mathrm{mV} \mathrm{s}^{-1}$.

Also, in a potential window from $\mathrm{Hg}^{2+}(0.84$ to $0.9 \mathrm{~V}) \mathrm{Ag}^{2+}(0.31-0.39 \mathrm{~V})$ and the use of the platinum nanoelectrode using the OSWV system (Figure 5 and 6) the $\mathrm{Hg}^{2+}$ and $\mathrm{Ag}^{2+}$ solutions of different concentration were analyzed.

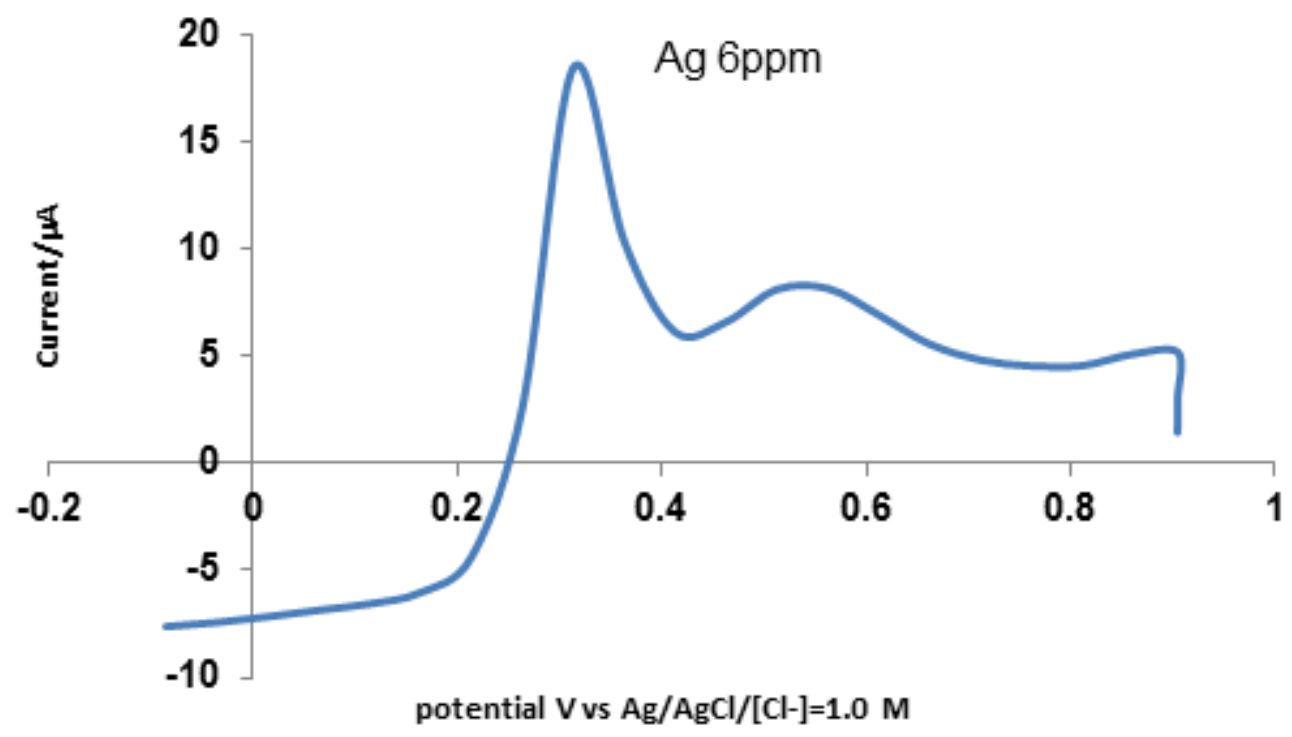


Figure 5 ; Cyclic voltammograms of $\mathrm{Ag}^{2+}$ solutions with 6 ppm concentration using the Platinum nanoelectrode at a scan rate of $50 \mathrm{mV} \mathrm{s}^{-1}$.

The reaction of the electrode to $\mathrm{Ag}^{2+}$ can be observed as being more active than the detection limit $\left(\mathrm{Cu}^{2+}\right.$ and $\left.\mathrm{Hg}^{2+}\right)$ of $8 \mathrm{nM}$. Figure 6 indicates the trajectory of peak current versus concentrations of $\mathrm{Hg}^{2+}, \mathrm{Cu}^{2+}$ and $\mathrm{Ag}^{2+}$.

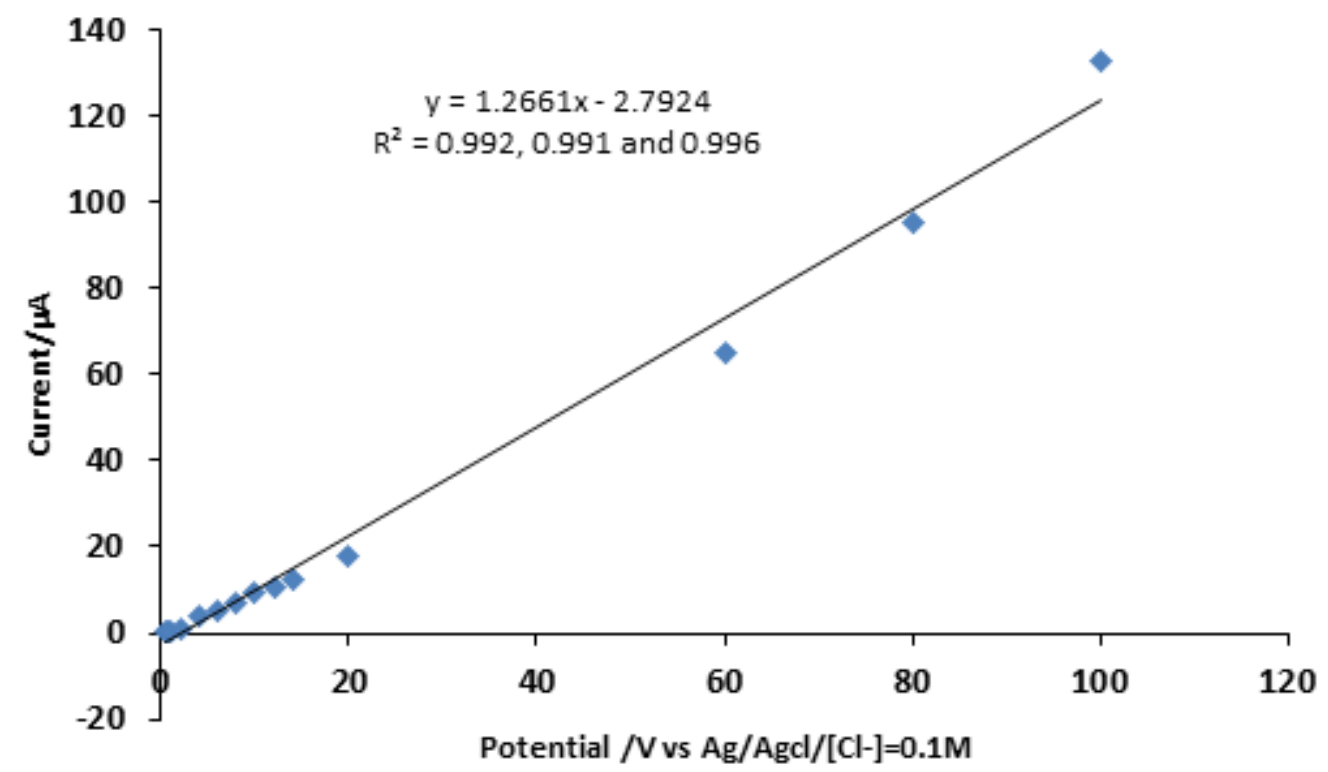

Figure 6: Plot peak current versus concentrations of $\mathrm{Hg}^{2+}, \mathrm{Cu}^{2+}$ and $\mathrm{Ag}^{2+}$ at a scan rate of $50 \mathrm{mV} \mathrm{s}^{-1}$.

The prepared electrode was observed to range from 0.009-4 M, 0.005-3 $\mathrm{M}$ and 0.003-1 M respectively to a linear detection of $0.992,0.991$ and 0.996 . In contrast, other sensors have previously recorded the detection efficiency of $\mathrm{Hg}^{2+}, \mathrm{Cu}^{2+}$ and $\mathrm{Ag}^{2+}$. It is clear that the range of detection of our research is superior to other studies, but comparable to some other tests. Platinum nanoparticles electrode stability was tested for 7 days, 14 days and 40 days respectively while keeping the electrodes at room temperature. Platinum nanoelectrode OSWVs have been reported in the ammonium acetate buffer $(\mathrm{pH}$ = 5.0) with $2.5 \mathrm{M} \mathrm{Hg}^{2+}, \mathrm{Cu}^{2+}$ and $\mathrm{Ag}^{2+}$ solutions. In the first two weeks, as seen in Figure 6 , just 4.1 percent was deteriorated. After 40 days, the sensitivity of the electrode was still greater than $82.1 \%$ [23]. 


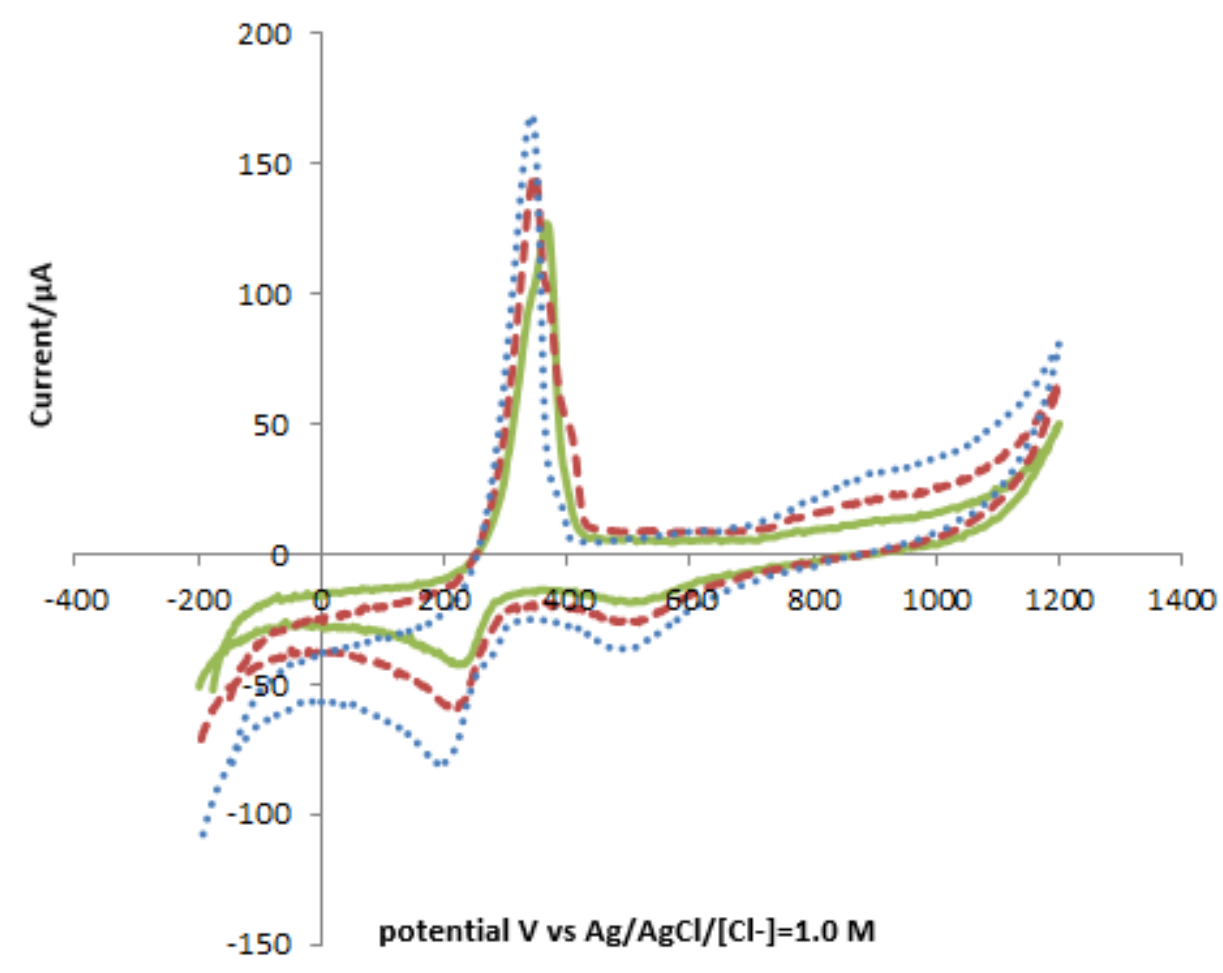

Figure 7: OSWVs of $2.5 \mathrm{M} \mathrm{Ag}^{2+}$ ammonium acetate solution using the platinum nanoelectrode with different days.

Ultrasound therapy has also been tested for the stability of platinum nanoelectrode. The sensitivity of $\mathrm{Hg}^{2+}, \mathrm{Cu}^{2+}$ and $\mathrm{Ag}^{2+}$ to detect also exceeded $76.6 \%$ by $82.4 \%$ and $92.6 \%$ after ultrasonic therapy in the clean ammonium buffer $(\mathrm{pH}=5.0)$, for $10 \mathrm{~min}$ [24]. The platinum nanoparticles were found to be solid enough for replicable study in tantalum surface electrodes. 


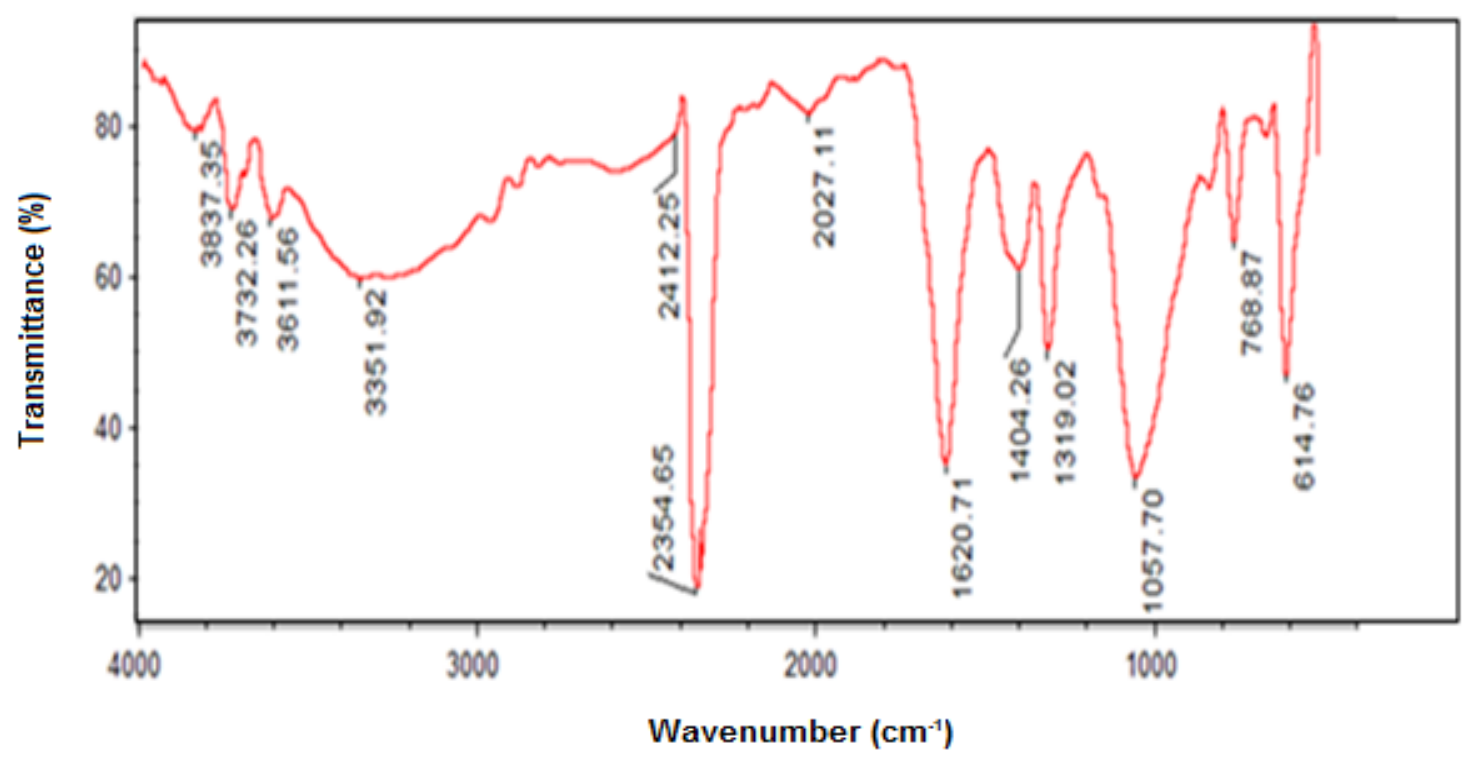

Figure 8: FT-IR spectrum of $\mathrm{Hg}^{2+}, \mathrm{Ag}^{+}$and $\mathrm{Cu}^{2+}$

To determine the nature of the sample between surface of electrode and $\mathrm{Hg}^{2+}, \mathrm{Ag}^{+}$ and $\mathrm{Cu}^{2+}$, FT-IR spectra of platinum nanoparticle electrode was recorded in the presence of $\mathrm{Hg}^{2+}, \mathrm{Ag}^{+}$and $\mathrm{Cu}^{2+}$ (Figure 8). The findings showed that the metal ion $\mathrm{Hg}^{2+}, \mathrm{Ag}^{+}$and $\mathrm{Cu}^{2+}$ was in perfect harmony with the nanoparticulate surface $\mathrm{Pt}$, and the literature published [25].

\section{Conclusions}

In summary, a new method for the manufacture of platinum nanoparticle electrode with a tuning thickness has been successfully produced through ion beam sputters and chemical corrosion. The heavy-metal ion measurement sensitivity was proportional to the pore electrode's thickness and completely saturated after $50 \mathrm{~nm}$. With the added thickness of the porous electrode, the analytical exposure to heavy metal ions is improved. Of $\mathrm{Cu}^{2+}, 0.005-3 \mathrm{M}$ for the $\mathrm{Ag}^{2+}$ analysis and $0.009-4 \mathrm{M}$ for the $\mathrm{Hg}^{2+}$, the linear detection range is $0.003-1 \mathrm{M}$. Such electrodes were also stable, reusable and antiinterfering.

\section{References}


1- Xia, Fangquan, Xin Zhang, Changli Zhou, Danzi Sun, Yanmin Dong, and Zhen Liu. "Simultaneous Determination of Copper, Lead, and Cadmium at Hexagonal Mesoporous Silica Immobilized Quercetin Modified Carbon Paste Electrode." Journal of Automated Methods and Management in Chemistry 2010 (2010): 1-6. https://doi.org/10.1155/2010/824197.

2- Altweiq, Abdelmnim, and Alkhawaldeh, Ahmad "The Determination of Some Heavy Metals in Different Selected Diets". Eurasian Journal of Analytical Chemistry 14 no. 4 (2019): emEJAC-00326.

3- Zhang, Xiao-Lei, Jennifer L Mcglothan, Omid Miry, Kirstie H Stansfield, Meredith K Loth, Patric K Stanton, and Tomás R Guilarte. "From the Cover: 7,8-Dihydroxyflavone Rescues Lead-Induced Impairment of Vesicular Release: A Novel Therapeutic Approach for Lead Intoxicated Children." Toxicological Sciences 161, no. 1 (April 2017): 186-95. https://doi.org/10.1093/toxsci/kfx210.

4- Shi, Yibing, Roumin Wang, Wei Yuan, Qingyun Liu, Mei Shi, Wei Feng, Zhiying Wu, $\mathrm{Ke} \mathrm{Hu}$, and Fuyou Li. "Easy-to-Use Colorimetric Cyanine Probe for the Detection of Cu2 in Wilson's Disease.” ACS Applied Materials \& Interfaces 10, no. 24 (2018): 20377-86. https://doi.org/10.1021/acsami.8b07081.

5- Chaney, Rufus L., Carrie E. Green, and Steven J. Lehotay. "Inter-Laboratory Validation of an Inexpensive Streamlined Method to Measure Inorganic Arsenic in Rice Grain." Analytical and Bioanalytical Chemistry 410, no. 22 (April 2018): 5703-10. https://doi.org/10.1007/s00216-018-1075-x.

6- Terol, Amanda, Eduardo Paredes, Salvador E. Maestre, Soledad Prats, and José L. Todolí. "Alcohol and Metal Determination in Alcoholic Beverages through HighTemperature Liquid-Chromatography Coupled to an Inductively Coupled Plasma Atomic Emission Spectrometer." Journal of Chromatography A 1218, no. 22 (2011): 3439-46. https://doi.org/10.1016/j.chroma.2011.03.060.

7- Martínez, David, Daniel Torregrosa, Guillermo Grindlay, Luis Gras, and Juan Mora. "Coupling Dispersive Liquid-Liquid Microextraction to Inductively Coupled Plasma Atomic Emission Spectrometry: An Oxymoron?” Talanta 176 (2018): 374-81. https://doi.org/10.1016/j.talanta.2017.08.036.

8- Premathilake, Dilshan, Ronald A. Outlaw, Samuel G. Parler, Sue M. Butler, and John R. Miller. "Electric Double Layer Capacitors for Ac Filtering Made from Vertically Oriented Graphene Nanosheets on Aluminum." Carbon 111 (2017): 231-37. https://doi.org/10.1016/j.carbon.2016.09.080. 
9- Wang, Zhong-Xia, and Shou-Nian Ding. “One-Pot Green Synthesis of High Quantum Yield Oxygen-Doped, Nitrogen-Rich, Photoluminescent Polymer Carbon Nanoribbons as an Effective Fluorescent Sensing Platform for Sensitive and Selective Detection of Silver(I) and Mercury(II) Ions." Analytical Chemistry 86, no. 15 (September 2014): 7436-45. https://doi.org/10.1021/ac501085d.

10- Hourani, Mohammed Khair, and Alkawaldeh, Ahmad. "Synergistic Effects of Bismuth Adatoms on Electrocatalytic Properties of Electrodeposited Nanostructured Platinum Electrodes." International Journal of Electrochemical Science, 2016, 3555-66. https://doi.org/10.20964/110434.

11- Zarei, K., and A. Khodadadi. "Very Sensitive Electrochemical Determination of Diuron on Glassy Carbon Electrode Modified with Reduced Graphene Oxide-Gold Nanoparticle-Nafion Composite Film." Ecotoxicology and Environmental Safety 144 (2017): 171-77. https://doi.org/10.1016/j.ecoenv.2017.06.030.

12- Park, Kyungtae, Daheui Choi, and Jinkee Hong. "Nanostructured Polymer Thin Films Fabricated with Brush-Based Layer-by-Layer Self-Assembly for Site-Selective Construction and Drug Release." Scientific Reports 8, no. 1 (2018). https://doi.org/10.1038/s41598-018-21493-9.

13- Saito, Mikiko, Jun Mizuno, Shunichi Koga, and Hiroshi Nishikawa. "Control for AuAg Nanoporous Structure by Electrodeposition and Dealloying." 2018 7th Electronic $\begin{array}{llll}\text { System-Integration } \quad \text { Technology } & \text { Conference } & \text { (ESTC), } & \end{array}$ https://doi.org/10.1109/estc.2018.8546350.

14- Chiu, Tai-Chia, and Shu-Wei Huang. "Synthesis of Fluorescent Carbon Nanoparticles as Selective and Sensitive Probes for Copper Ions." Journal of Biosensors \& Bioelectronics 09 (2018). https://doi.org/10.4172/2155-6210-c1-037.

15- Wang, Shaopeng, Erica S. Forzani, and Nongjian Tao. "Detection of Heavy Metal Ions in Water by High-Resolution Surface Plasmon Resonance Spectroscopy Combined with Anodic Stripping Voltammetry." Analytical Chemistry 79, no. 12 (2007): 4427-32. https://doi.org/10.1021/ac0621773.

16- Gao, Chao, Xin-Yao Yu, Shi-Quan Xiong, Jin-Huai Liu, and Xing-Jiu Huang. "Electrochemical Detection of Arsenic(III) Completely Free from Noble Metal: Fe3O4 Microspheres-Room Temperature Ionic Liquid Composite Showing Better Performance than Gold." Analytical Chemistry 85, no. 5 (2013): 2673-80. https://doi.org/10.1021/ac303143x. 
17- Cui, Lin, Jie Wu, and Huangxian Ju. "Electrochemical Sensing of Heavy Metal Ions with Inorganic, Organic and Bio-Materials." Biosensors and Bioelectronics 63 (2015): 276-86. https://doi.org/10.1016/j.bios.2014.07.052.

18- Xiao, Deli, Siqiao Li, Shubo Liu, Hua He, and Jinrong Lu. “One-Step Hydrothermal Synthesis of Photoluminescent Carbon Nitride Dots Derived from Ionic Liquids." New Journal of Chemistry 40, no. 1 (2016): 320-24. https://doi.org/10.1039/c5nj01717c.

19- Sakiew, Wjatscheslaw, Stefan Schrameyer, Marco Jupé, Philippe Schwerdtner, Nick Erhart, Kai Starke, and Detlev Ristau. "Influence of Ion Beam Parameters onto TwoDimensional Optical Thin Film Thickness Distributions Deposited by Ion Beam Sputtering." Thin Solid Films $682 \quad$ (2019): $109-20$. https://doi.org/10.1016/j.tsf.2019.05.027.

20- Abu-Ali, Hisham, Alexei Nabok, Thomas J. Smith, and Maythem Al-Shanawa. "Development of a Novel Electrochemical Inhibition Sensor Array Based on Bacteria Immobilized on Modified Screen-Printed Gold Electrodes for Water Pollution Detection," 2018. https://doi.org/10.20944/preprints201807.0351.v1.

21- Alkhawaldeh, Ahmad Khalaf, Monzer M.Krishan, Abdelmnim Altwaiq, and Reem Naser Dabaibeh. "Preparation of Nanostructured/ Microplatinum Surfaces by Application of a Square Wave Potential Regime for Methanol Oxidation". Eurasian Journal of Analytical Chemistry 15 no. 1 (2020): emEJAC-00362.

22- Cui, Liang, Jingquan Liu, Rui Wang, Zhen Liu, and Wenrong Yang. "A Facile 'Graft from' Method to Prepare Molecular-Level Dispersed Graphene-Polymer Composites." Journal of Polymer Science Part A: Polymer Chemistry 50, no. 21 (March 2012): 442332. https://doi.org/10.1002/pola.26264.

23- Pandey, Nidhi, Mukul Gupta, and D. M. Phase. "Preparation and Characterization of Fe4N Thin Film Deposited by High Power Impulse Magnetron Sputtering." Dae Solid State Physics Symposium 2018, 2019. https://doi.org/10.1063/1.5113132.

24- Alshamaileh, Ehab, Mazen Al-Sulaibi, Ahmad Al-Khawaldeh, Mansour H. Almatarneh, Dina El-Sabawi, and Aiman Al-Rawajfeh. "Current Status of Nanotechnology in Jordan." World Journal of Science, Technology and Sustainable Development 13, no. 2 (April 2016): 66-81. https://doi.org/10.1108/wjstsd-01-2016$\underline{0001 .}$.

25- Zhang, Shanshan, Xingxing Wu, Qingfen Niu, Zongrang Guo, Tianduo Li, and Haixia Liu. "Highly Selective and Sensitive Colorimetric and Fluorescent Chemosensor for Rapid Detection of $\mathrm{Ag}+, \mathrm{Cu} 2+$ and $\mathrm{Hg} 2+$ Based on a Simple Schiff Base." Journal of Fluorescence 27, no. 2 (2016): 729-37. https://doi.org/10.1007/s10895-016-2005-y. 
